\title{
NEIGHBORHOOD EXTENSIONS OF CONTINUOUS SELECTIONS
}

\author{
M. K. FORT, JR. ${ }^{1}$
}

Hanner (see [2, Theorem 19.2]) has proved that the property of being a neighborhood extension space with respect to the class of paracompact spaces is a local property. Theorem 1 of this paper generalizes Hanner's result, and states roughly that if a continuous selection can be locally extended to a continuous selection, then it has a neighborhood extension which is also a continuous selection. The proof of Theorem 1 differs considerably from Hanner's proof, and seems at least as simple.

Let $X$ be a paracompact space and $Y$ a topological space. We assume that for each $x \in X, F(x)$ is a subset of $Y$. If $K$ is a subset of $X$ and $\phi$ is a mapping (continuous function) on $K$ into $Y$, then $\phi$ is a selection for $F$ if $\phi(x) \in F(x)$ for each $x \in K$. A subset $S_{1}$ of $Y$ is an $F$-neighborhood extension set with respect to a subset $S_{2}$ of $X$ if each mapping $\phi: C \rightarrow S_{1}, \phi$ a selection for $F, C \subset S_{2}$ and $C$ closed relative to $S_{2}$, can be extended to a mapping $\psi: G \rightarrow S_{1}$, where $\psi$ is a selection for $F, G \subset S_{2}$ and $G$ is open relative to $S_{2}$.

We assume that $A$ is a closed subset of $X$ and that $f$ is a selection for $F$ which maps $A$ into $Y$.

THEOREM 1. If for each $x \in A$ there exist neighborhoods $V_{x}$ of $x$ and $U_{x}$ of $f(x)$ such that $U_{x}$ is an $F$-neighborhood extension set with respect to $\bar{V}_{x}$, and $f\left[\bar{V}_{x} \cap A\right] \subset U_{x}$, then there exists an open set $N \supset A$ and $a$ mapping $g: N \rightarrow Y$ such that $g \mid A=f$ and $g$ is a selection for $F$.

Proof. Let $g_{1}=\left\{W \mid W=X-A\right.$ or $W=V_{x}$ for some $\left.x \in A\right\}$. Since $X$ is paracompact, there is a $\sigma$-discrete refinement $\mathcal{S}_{2}$ of $\mathcal{G}_{1}$. We may assume without loss of generality that $\left\{\bar{W} \mid W \in \mathrm{g}_{2}\right\}$ is also a refinement of $\mathcal{G}_{1}$. We let $R=\left\{W \mid W \in \mathcal{G}_{2}\right.$ and $\left.W \cap A \neq \varnothing\right\}$. It is possible to represent $R=\bigcup_{n=1}^{\infty} R_{n}$, where each $R_{n}$ is a discrete collection of open sets.

We wish to define inductively a sequence $A_{0} \subset A_{1} \subset A_{2} \subset \cdots$ of closed sets and mappings $g_{k}: A_{k} \rightarrow Y$ such that:

(i) $A_{0}=A$ and $g_{0}=g$,

(ii) if $W \in \mathcal{R}_{k}$, then $W \cap A$ is contained in the interior of $A_{k+1}$,

Presented to the Society November 21, 1959, under the title $A$ lifting and neighborhood extension theorem for mappings into fiber spaces; received by the editors January 25, 1960.

1 The author is an Alfred P. Sloan Research Fellow. This work was also partially supported by a National Science Foundation Grant NSF G-3016. 
(iii) each $g_{k}$ is a selection for $F$ and $g_{k+1} \mid A_{k}=g_{k}$ for $k=0,1,2, \ldots$. We define $A_{0}$ and $g_{0}$ so as to satisfy (i). Now let us assume that $A_{0} \subset A_{1} \subset \cdots \subset A_{n}$ and $g_{0}, g_{1}, \cdots, g_{n}$ have been defined so as to satisfy the above conditions. We must define $A_{n+1}$ and $g_{n+1}$.

Let $W$ be any member of $\Omega_{n+1}$. There exists $x \in A$ such that $\bar{W} \subset V_{x}$. The set $g_{n}^{-1}\left[U_{x}\right]$ is open relative to $A_{n}$, and thus there exists an open set $W_{1}$ such that $g_{n}^{-1}\left[U_{x}\right]=A_{n} \cap W_{1}$. Since $g_{n}[\bar{W} \cap A]$ $=f[\bar{W} \cap A] \subset f\left[\bar{V}_{x} \cap A\right] \subset U_{x}$, it follows that $\bar{W} \cap A \subset W_{1}$. By normality of $X$, there exists an open set $W_{2}$ such that $\bar{W} \cap A \subset W_{2}$ and $\bar{W}_{2} \subset W_{1}$. If we define $W_{3}=W \cap W_{2}$, then $g_{n}\left[\bar{W}_{3} \cap A_{n}\right] \subset U_{x}$ and we may extend $g_{n} \mid\left(\bar{W}_{3} \cap A_{n}\right)$ to a mapping $\psi_{W}: W_{4} \rightarrow U_{x}$, where $W_{4}$ is an open subset of $\bar{V}_{x}$ and $\psi_{W}$ is a selection for $F$. There exists an open set $W_{5}$ such that $\bar{W}_{3} \cap A_{n} \subset W_{5}$ and $\bar{W}_{5} \subset W_{4}$.

We now define

$$
A_{n+1}=A_{n} \cup \underset{W \in \mathbb{R}_{n}}{\bigcup}\left(\bar{W}_{5} \cap \bar{W}_{3}\right),
$$

and

$$
g_{n+1}(x)= \begin{cases}g_{n}(x), & \text { for } x \in A_{n}, \\ \psi_{W}(x), & \text { for } x \in \bar{W}_{5} \cap \bar{W}_{3} \text { for some } W \in Q_{n} .\end{cases}
$$

The fact that $R_{n}$ is discrete implies that the set $A_{n+1}$ is closed. It is easy to see that for each $W \in \mathcal{R}_{n}, W \cap A \subset W_{3} \cap W_{5}$ and hence $W \cap A$ is contained in the interior of $A_{n+1}$. The function $g_{n+1}$ is well defined, and its continuity follows from the fact that $R_{n}$ is a discrete collection of open sets. It is also obvious that $g_{n+1}$ is a selection for $F$.

We now obtain $N$ and $g$ by letting $N=\cup_{n=1}^{\infty}$ (interior $A_{n}$ ), and defining $g(x)=g_{n}(x)$ for $x \in$ (interior of $A_{n}$ ).

The preceding theorem is of interest in connection with the problem $\left(Q_{2}\right)$ stated by Michael in [4]. The next theorem, which is a combination lifting and extension theorem, is of the same general nature as Steenrod's existence theorem on cross sections (see [5, p. 55]). A special case of this result was used by the author in [1].

We now let $Y$ be a fiber space (in the sense of Hilton, see [3, p. 46]) with base space $B$, fiber $K$, and projection $p: Y \rightarrow B$. It is again assumed that $X$ is a paracompact space and that $A$ is a closed subset of $X$. We assume mappings $f: A \rightarrow Y$ and $h: X \rightarrow B$ such that $p f=h \mid A$. The definition of ANR (absolute neighborhood retract) is given in [2].

Theorem 2. If $K$ is an $A N R$, then there exists an open set $N \supset A$ and a mapping $g: N \rightarrow Y$ such that $g \mid A=f$ and $p g=h \mid N$. 
Proof. We define $F(x)=p^{-1} h(x)$ for each $x \in X$. For each $x \in A$, let $W_{x}$ be a neighborhood of $h(x)$ such that there exists a homeomorphism $\eta_{x}$ on $W_{x} \times K$ onto $p^{-1}\left[W_{x}\right]$ for which $p \eta_{x}(b, k)=b$. We define $U_{x}=Y$, and choose $V_{x}$ to be any neighborhood of $x$ for which $h\left[\bar{V}_{x}\right] \subset W_{x}$.

We will show that each $U_{x}$ is an $F$-neighborhood extension set with respect to $\bar{V}_{x}$. Thus, we consider a closed subset $C$ of $\bar{V}_{x}$ and a selection for $F, \phi: C \rightarrow U_{x}$. The mapping $\eta_{x}^{-1} \phi$ maps $C$ into $W_{x} \times K$, and hence we can represent $\eta_{x}^{-1} \phi(t)=(\alpha(t), \beta(t))$, where $\alpha$ and $\beta$ are mappings on $C$ into $W_{x}$ and $K$ respectively. Since $K$ is an ANR, and $\bar{V}_{x}$ is normal we can extend $\beta$ to a mapping $\beta^{\prime}: G_{1} \rightarrow K$, where $G_{1}$ is open relative to $\bar{V}_{x}$. Since $\alpha(t)=p \eta_{x}(\alpha(t), \beta(t))=p \eta_{x} \eta_{x}^{-1} \phi(t)=p \phi(t)=h(t)$ for $t \in C$, we can let $G_{2}=\bar{V}_{x} \cap h^{-1}\left[W_{x}\right]$ and extend $\alpha$ to $a^{\prime}: G_{2} \rightarrow W_{x}$ by letting $\alpha^{\prime}=h \mid G_{2}$. We now obtain the desired selection for $F$ by letting $G=G_{1} \cap G_{2}$ and defining

$$
\psi(t)=\eta_{x}\left(\left(\alpha^{\prime}(t), \beta^{\prime}(t)\right)\right),
$$

for $t \in G$.

It is now easy to verify that the hypotheses of Theorem 1 are satisfied. Thus, there exists an open set $N \supset A$ and a mapping $g: N \rightarrow Y$ such that $g \mid A=f$ and $g$ is a selection for $F$. The fact that $g$ is a selection for $F$, however, means that $p g=h \mid N$, and this concludes the proof of Theorem 2 .

A space $Y$ has the neighborhood extension property with respect to the class of all paracompact spaces if corresponding to each paracompact space $X$ and mapping $f$ of a closed subset of $X$ into $Y$ there is an extension of $f$ whose domain is an open set. We let $M$ be the set of all spaces $Y$ which have the neighborhood extension property with respect to the class of all paracompact spaces. The following theorem, which we prove by applying Theorem 1 , seems to have been first demonstrated by Hanner (see [2, p. 340]).

TheOREM 3. If each point in a space $Y$ has a neighborhood which belongs to $M$, then $Y$ belongs to $M$.

Proof. Let $X$ be a paracompact space, let $A$ be a closed subset of $X$, and let $f$ be a mapping of $A$ into $Y$.

We define $F(x)=Y$ for each $x \in A$. For each $x \in A$, we choose a neighborhood $U_{x}$ of $f(x)$ such that $U_{x} \in M$. We may now choose a neighborhood $V_{x}$ of $x$ such that $f\left[\bar{V}_{x} \cap A\right] \subset U_{x}$. Since $\bar{V}_{x}$ is a paracompact space and $U_{x} \in M$, it follows that $U_{x}$ is an $F$-neighborhood extension set with respect to $\bar{V}_{x}$. Thus, by Theorem 1 we can extend $f$ to a mapping whose domain is an open set. This proves that $Y \in M$. 


\section{REFERENCES}

1. M. K. Fort, Jr., Images of plane continua, Amer. J. Math. vol. 81 (1959) pp. 541-546.

2. O. Hanner, Retraction and extension of mappings of metric and non-metric spaces, Ark. Mat. vol. 2 (1954) pp. 315-360.

3. P. J. Hilton, An introduction to homotopy theory, Cambridge Tracts in Mathematics and Mathematical Physics, no. 43, Cambridge University Press, 1953.

4. Ernest Michael, Continuous selections. II, Ann. of Math. vol. 64 (1956) pp. $562-580$. 1951.

5. Norman Steenrod, The topology of fibre bundles, Princeton University Press,

The University of Georgia

\section{A REMARK ON THE CHARACTERIZATION OF HOMOTHETIC TRANSFORMATION AND INVERSION ${ }^{1}$}

\section{CHIN-SHUI HSÜ}

Given two closed orientable $C^{2}$ surfaces $S, \bar{S}$ in $E^{3}$, let $h: S \rightarrow \bar{S}$ be a differentiable homeomorphism, such that referring to a suitable origin $O$

$$
\bar{X}=k X,
$$

with $k$ nonzero and $C^{2}$ at points at which $X \neq 0, \bar{X} \neq 0$. Write $p=-X \cdot N, \bar{p}=-\bar{X} \cdot \bar{N}$. Suppose further that $S, \bar{S}$ contains no pieces of cones with vertex $O$. We use the form $b=(\bar{N} \times X) \cdot d X$, introduced in [1]. We generalize results of [1] to

THEOREM $1^{\prime}$. If $\iint_{S}(H \mp k \bar{H}) p d A=0$, then $k=$ const., i.e. the map $h$ is a homothetic transformation with center $O$.

Proof. The formulae $p d \bar{A}=k^{3} p d A$ and $d b=\left(2 / k^{2}\right) \bar{H} d \bar{A}+2 \bar{N} \cdot N d A$ yield $\iint_{S} N \cdot \bar{N} d A=-\iint_{S} k \bar{H} p d A$. Combining with $\iint_{S} d A=\iint_{S} H p d A$ gives

$$
\iint_{S}(1 \mp N \cdot \bar{N}) d A=\iint_{S}(H \pm k \bar{H}) p d A,
$$

and the theorem follows as in [1].

Received by the editors May 15, 1959.

1 The author thanks Professor Hans Samelson for his suggestions for revision. 